\title{
Modulation of Neural Activity during Observational Learning of Actions and Their Sequential Orders
}

\author{
Scott H. Frey and Valerie E. Gerry \\ Lewis Center for Neuroimaging and Department of Psychology, University of Oregon, Eugene, Oregon 97403-1227
}

\begin{abstract}
How does the brain transform perceptual representations of others' actions into motor representations that can be used to guide behavior? Here we used functional magnetic resonance imaging to record human brain activity while subjects watched others construct multipart objects under varied task demands. We find that relative to resting baseline, passive action observation increases activity within inferior frontal and parietal cortices implicated in action encoding (mirror system) and throughout a distributed network of areas involved in motor representation, including dorsal premotor cortex, pre-supplementary motor area, cerebellum, and basal ganglia (experiments 1 and 2). Relative to passive observation, these same areas show increased activity when subjects observe with the intention to subsequently reproduce component actions using the demonstrated sequential procedures (experiment 1 ). Observing the same actions with the intention of reproducing component actions, but without the requirement to use the demonstrated sequential procedure, increases activity in the same regions, although to a lesser degree (experiment 2). These findings demonstrate that when attempting to learn behaviors through observation, the observers' intentions modulate responses in a widely distributed network of cortical and subcortical regions implicated previously in action encoding and/or motor representation. Among these regions, only activity within the right intraparietal sulcus predicts the accuracy with which observed procedures are subsequently performed. Successful formation of motor representations of sequential procedures through observational learning is dependent on computations implemented within this parietal region.
\end{abstract}

Key words: observational learning; sequence representation; fMRI; intraparietal sulcus; mirror system; rehabilitation

\section{Introduction}

Observational learning plays an important role in acquiring motor skills throughout the human lifespan (Carroll and Bandura, 1982; Blandin, 2002; Heyes and Foster, 2002). Understanding the brain mechanisms involved may facilitate development of techniques to improve acquisition or rehabilitation of motor skills. At present, however, very little is known about this form of skill learning, which is distinguished by the absence of immediate sensory feedback.

Recent findings suggest that observation of others' actions may be sufficient to induce the formation of new motor traces in primary motor cortex (M1). Repeated observation of repetitive thumb extension modifies the direction of thumb movements evoked subsequently by transcranial magnetic stimulation over M1 (Stefan et al., 2005; Celnik et al., 2006). These changes reflect the acquisition of kinematically precise and effector-specific motor representations and may be driven by input from the mirror system via direct connections between ventral premotor cortex and M1 motoneurons (Cerri et al., 2003).

Received June 29, 2006; revised Nov. 6, 2006; accepted Nov. 7, 2006.

This work was supported by grants from the National Institute of Mental Health (K01 MH002022-01) and the James S. McDonnell Foundation to S.H.F. We thank Dr. Michael Posner, Dr. Claudia Danielmeier, and anonymous reviewers for helpful comments on previous versions of this manuscript.

Correspondence should be addressed to Scott H. Frey, Lewis Center for Neuroimaging and Department of Psychology, 1227 Department of Psychology, University of Oregon, Eugene, OR 97403-1227. E-mail: shfrey@uoregon.edu.

DOI:10.1523/JNEUROSCI.3914-06.2006

Copyright $\odot 2006$ Society for Neuroscience $\quad$ 0270-6474/06/2613194-08\$15.00/0
Single neurons within the ventral premotor cortex [area F5c (Rizzolatti et al., 1988)] and the rostral inferior parietal lobule [area PF (Fogassi et al., 2005)] of macaques are activated selectively either by the execution or observation of the same actions. Importantly, these "mirror" neurons are part of a larger representational system that may support a "vocabulary" of hand actions (e.g., grasping, holding, twisting) from which complex manual actions are assembled (Rizzolatti et al., 1988; Gallese et al., 1996). In humans, similar responses are present within inferior frontal (pars opercularis, pars triangularis, and ventral precentral gyrus) and anterior inferior parietal (supramarginal gyrus) cortices when passively observing dynamic (Grafton et al., 1996) or static (Johnson-Frey et al., 2003) hand-object interactions, and activity increases when watching with the intention to imitate subsequently (Grezes et al., 1999; Iacoboni et al., 1999; Hermsdörfer et al., 2001; Koski et al., 2003; Buccino et al., 2004). Areas displaying these mirroring responses may provide a mechanism for mapping between the observed behaviors of others and our own internal motor representations (Rizzolatti and Craighero, 2004).

Together, these findings implicate the parietofrontal mirror system in encoding the spatial components of observed actions and $\mathrm{M} 1$ in the formation of novel motor memories through observation. Yet, what mechanisms are involved in constructing temporal representations of observed action components? Successful performance of many skills demands that actions necessary to achieve specific subgoals are executed in the proper serial 


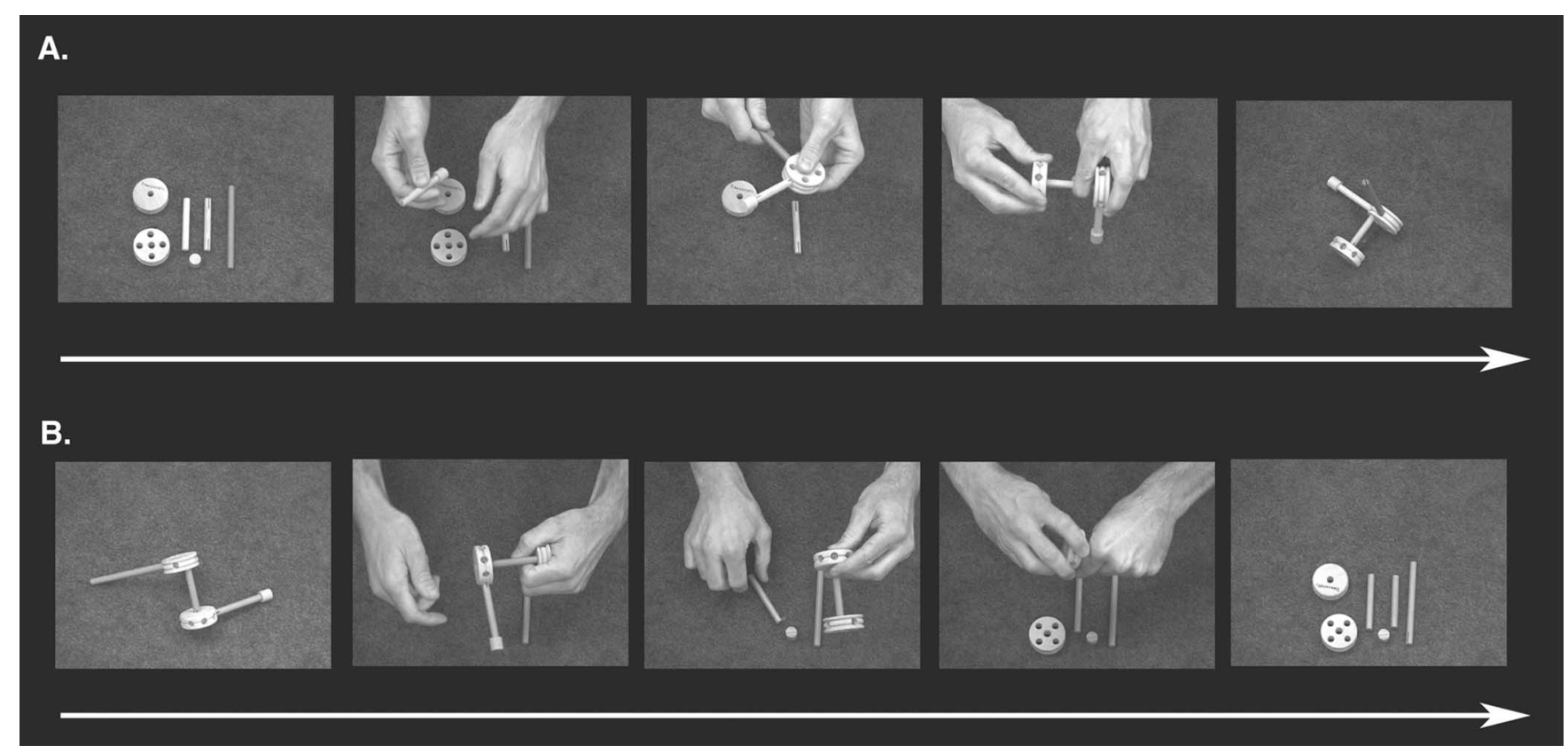

Figure 1. Sample stimuli and the object assembly task. Subjects watched video clips of objects being assembled from a set of six parts. $A$, Learn condition clips began with parts in a standard arrangement and ended with a completed object. $\boldsymbol{B}$, Perceptual Control condition clips began with a fully assembled object and ended with the disassembled parts in the standard arrangement. The resting baseline condition is not shown.

order (Lashley, 1951). We predicted that learning the order of component actions that constitute an observed procedure would involve the well established motor sequence learning network, including dorsal premotor cortex (PMd), inferior parietal and mesial frontal [supplementary motor area (SMA) and/or preSMA] cortices, cerebellum, and basal ganglia (Doyon et al., 1996; Hazeltine et al., 1997; Grafton et al., 1998). We also asked whether activity within any of these areas during action encoding predicts the accuracy with which observed procedures are subsequently performed. A likely candidate is anterior intraparietal cortex, which is implicated both in forming effector-independent representations of motor sequences (Grafton et al., 1998) and in coding the goals of observed (Hamilton and Grafton, 2006) and executed (Tunik et al., 2005) grasping actions.

\section{Materials and Methods}

Experiment 1: learning of demonstrated component actions and their sequences for manual reproduction

Participants. Blood oxygen level-dependent (BOLD) magnetic resonance imaging (MRI) was used to record neural activity from the entire brain while 19 ( 13 males) healthy, college-aged adults observed digital videos of objects being assembled manually. All participants were right-handed as verified by the Edinburgh Handedness Inventory (Oldfield, 1971).

Procedure. Stimuli consisted of four $24 \mathrm{~s}$ duration videos with a superimposed central fixation cross. Four Learn condition videos each show a different Tinkertoy (Hasbro) object being constructed from the same six parts (Fig. 1A).

Each Learn condition video begins with the six parts laid out in the same configuration on a table surface (i.e., standard configuration) (Fig. $1 A)$ and ends with the completed object resting by itself on the table surface for 2-3 s. A fifth Perceptual Control video shows a different object, created from the same parts, being disassembled manually (Fig. $1 B$ ). The Perceptual Control video begins with a completed object resting on the table surface (Fig. $1 B$ ) and ends with the six parts laid out on the table in the standard configuration for $2-3 \mathrm{~s}$. Object configurations were designed to avoid duplication. The sequence in which parts were joined to form each object was determined pseudo-randomly. Each part was assigned a number, and a random-number generator was used to create separate lists prescribing the sequential orders in which parts would be joined when creating each object configuration. In cases in which a given part could not be joined to the existing configuration as a result of physical constraints, the next part indicated on the random list was selected. This was repeated until all parts were unified into a single object. To discourage encoding strategies that rely on the relative leftright locations of object parts, within each run of the experiment videos in both conditions were shown in two orientations rotated by $180^{\circ}$ (i.e., hand movements directed toward vs away from the observer). Video orientation was counterbalanced such that toward and away orientations occurred with equal frequency across functional runs. All participants viewed the same stimulus videos in counterbalanced order. Stimulus delivery was digitally synchronized with the MRI scanner using Presentation software. Every participant completed eight $252 \mathrm{~s}$ functional runs, the order of which was counterbalanced across individuals. Each run consisted of three repetitions of the Learn condition video showing a single object being constructed and three repetitions of the Perceptual Control video. These conditions were presented in counterbalanced order across runs and were separated by $12 \mathrm{~s}$ rest (baseline) blocks consisting of a fixation cross displayed against a black screen.

Before each functional scan, subjects were instructed to watch each Learn condition video (object being constructed) so that they would be able to reconstruct the object using the exact sequence as the actor at the end of the run. For the Perceptual Control video (object being dismantled), they were instructed to simply watch the video but not to focus on remembering the actions performed, because they would not need to recollect or reproduce them.

At the end of each run, the subject was moved out of the MRI scanner. A Plexiglas board with the Tinkertoy pieces affixed by Velcro was brought into the scan room and held within the subject's reach. Participants remained supine on the bed of the scanner with their heads positioned in the radiofrequency coil. They were instructed to reconstruct the Learn condition object from the immediately preceding run using the exact sequence of actions observed, while keeping their heads as stationary as possible. Performances were videotaped, and the completed object was also filmed for off-line coding. The bed of the scanner was then returned to its original position in preparation for the next run. 
Experiment 2: intending to learn demonstrated component actions Only the instructions for the Learn condition were modified. Ten (six females) healthy, right-handed, college-aged participants were instructed to observe the videos of objects being constructed with the intention of recreating items manually at the end of the run. No mention was made of using any particular procedure when assembling the items. All other aspects of the design remained the same.

Behavioral data analyses. Video was scored off-line by two trained raters, one of whom was naive to the hypotheses being tested. Accuracy of the sequence with which objects were reconstructed was scored relative to the correct sequence for that object (i.e., the sequence demonstrated by the actor in the respective stimulus video). Coders recorded the order in which the subjects manually selected the parts from the Plexiglas board. If a subject picked up a piece and then returned it to the board (self correction), it was not counted as a part selection. Each individual action component (grasping and joining a single Tinkertoy part) was assigned 1 point for each part it was correctly selected ahead of and 1 point for each part it was correctly selected after. The total possible score for a six-piece sequence was therefore 30 points. Sequence scores were then normalized (demeaned) before inclusion in the functional MRI (fMRI) analyses by taking the average of all of the subjects' scores from all runs and subtracting this number from each individual score. There were two runs (of the total 152 runs) in which object assembly was not filmed because of technical error. In these two instances, missing data were replaced by the average scores computed across those subjects' seven other runs. Completed objects were scored as either correct or incorrect depending on whether their three-dimensional structure matched that of the corresponding stimulus object.

MRI procedure. All scans were performed on a Siemens (Erlangen, Germany) 3 tesla Allegra MRI scanner. High-resolution T1-weighted structural images were acquired using the three-dimensional MP-RAGE (multiplanar rapidly acquired gradient echo) pulse sequence: repetition time (TR), $2000 \mathrm{~ms}$; echo time (TE), $4.38 \mathrm{~ms}$; flip angle, $8.0^{\circ}$; $256 \times 176$ voxel matrix; field of view (FOV), $256 \mathrm{~mm}$; 176 contiguous axial slices; thickness, $1.0 \mathrm{~mm}$. Auto Align Scout and True FISP sequences were executed before the start of each functional run to ensure that slices were prescribed in exactly the same positions across runs. BOLD echoplanar images were collected using a T2 ${ }^{*}$-weighted gradient echo sequence: TR, $2000 \mathrm{~ms}$; TE, $30 \mathrm{~ms}$; flip angle, $80^{\circ}$; $64 \times 64$ voxel matrix; FOV, $200 \mathrm{~mm}$; 33 contiguous axial slices; thickness, $3.0 \mathrm{~mm}$. The initial four scans in each run were discarded to allow steady-state magnetization to be approached. Raw image data were reconstructed using two-dimensional fast Fourier transform with a distortion correction to reduce artifact attributable to magnetic field inhomogeneities and converted to NIFTI (Neuroimaging Informatics Technology Initiative) format using MRIConvert software (http://lcni.uoregon.edu/ jolinda/MRIConvert/). All data preprocessing and modeling were conducted with FSL version 3.2 (http://www.fmrib.ox.ac.uk/fsl/).

fMRI data analyses. Each fMRI run for a given subject was modeled separately at the first level. Before statistical estimation, the following preprocessing steps were undertaken: motion correction using MCFLIRT (Jenkinson et al., 2002); non-brain removal using BET (Smith, 2002); spatial smoothing using a Gaussian kernel of $5 \mathrm{~mm}$ (full-width at half-maximum); mean-based intensity normalization of all volumes by the same factor; high-pass temporal filtering $(\sigma=50.0 \mathrm{~s})$. Estimates of the degrees of freedom in the statistical model were corrected for autocorrelation in the data by using the FSL pre-whitening technique (Woolrich et al., 2001). Time-series statistical analysis was performed using FILM with local autocorrelation correction (Woolrich et al., 2001). Delays and undershoots in the hemodynamic response were accounted for by convolving the model with a double-gamma basis function. Registration to high-resolution and standard images (Montreal Neurological Institute template) was implemented using FLIRT (Jenkinson and Smith, 2001; Jenkinson et al., 2002). Inter-session (level 2), inter-subject (level 3 ), and inter-experiment (level 4) random-effects components of mixedeffects variance were modeled and estimated using FLAME Stage 1 (Beckmann et al., 2003). Z (Gaussianized T/F) statistic images were thresholded using clusters determined by $z>2.3$ unless otherwise indi- cated and a (corrected) cluster significance threshold of $p<0.05$ (Worsley et al., 1992). Verification of anatomical localization was undertaken by manual comparison with an atlas (Duvernoy, 1991) and overlaying activation maps of the population, landmark, and surfacebased atlas of Van Essen (2005) using CARET software (Van Essen et al., 2001). Data are displayed on a normalized, high-resolution template (http://www.jonaskaplan.com/atlases.html).

To investigate activity correlated with learning, an initial region of interest (ROI) was defined functionally as consisting of those voxels showing significantly greater activity in the Learn versus the Perceptual Control condition in the inter-subject (level 3) analysis described above. A model function was defined based on each subject's group normalized mean performance score on the object assembly task. This model was convolved with an estimate of the hemodynamic response function (double-gamma function) and fit to the contrast of the parameter estimate (cope) images for each subject resulting from inter-subject (level 3 ) comparison between the Learn condition and the resting baseline (modeled implicitly). Clusters with $Z>2.3$ and a corrected significance threshold of $p<0.05$ were determined to have activity correlated with performances on the object assembly task. The FSL Featquery was then used to compute mean contrast of parameter estimate values (copes) across all voxels within this functionally defined ROI separately for each subject $(n=19)$ and each session $(n=8)$ for the Learn condition versus the resting baseline comparison. A grand mean was then computed for each subject by averaging resulting values across sessions. Standard correlation analysis was then performed on each subject's grand mean versus their mean normalized performances on the object assembly task.

\section{Results}

\section{Experiment 1: effects of intending to learn demonstrated component actions and their sequential order}

Figure 2 shows that relative to the resting baseline, both passive observation (Fig. 2 A, Perceptual Control condition) and watching with the intention to learn action components and their demonstrated sequence (Fig. 2 B, Learn condition) are associated with increased bilateral activity within the parietofrontal mirror system (i.e., inferior frontal and parietal cortices) and in cortical and subcortical areas associated with motor learning, including PMd, basal ganglia, cerebellum, and pulvinar nucleus. The one exception is pre-SMA, which was only detected in the Learn condition (Fig. 2, compare $A, B$ ). Data were extracted from an ROI defined by the extent of the pre-SMA cluster observed in the Learn condition versus resting baseline contrast. Analysis confirms that pre-SMA does not differ from the resting baseline during passive action observation $\left(t_{(18)}<1.0\right)$. However, when observing with the intention to learn, pre-SMA activity is significantly greater than baseline $\left(t_{(18)}=8.0 ; p<0.0001\right)$ and also exceeds responses during passive action observation $\left(t_{(18)}=6.7 ; p<0.0001\right)$. Increased activity extending beyond parietal and inferior frontal cortices in response to passive observation was unanticipated and, as discussed below, suggests that mirroring may be a widely distributed property of the cortical and subcortical motor representation system.

Direct statistical comparison of the Learn versus the Perceptual Control condition reveals that observing actions with the intention to subsequently reproduce them results in increased activity throughout the parietofrontal mirror system (Fig. 3). This is consistent with previous observations in demonstrating that activity within this network is modulated top-down by the observers' intentions (Grezes et al., 1999; Iacoboni et al., 1999; Koski et al., 2003; Buccino et al., 2004). As predicted, activity is also increased throughout areas of the motor learning network including PMd, pre-SMA, basal ganglia (globus pallidus), cerebellum, and also hippocampus (Schendan et al., 2003) (Fig. 3). 


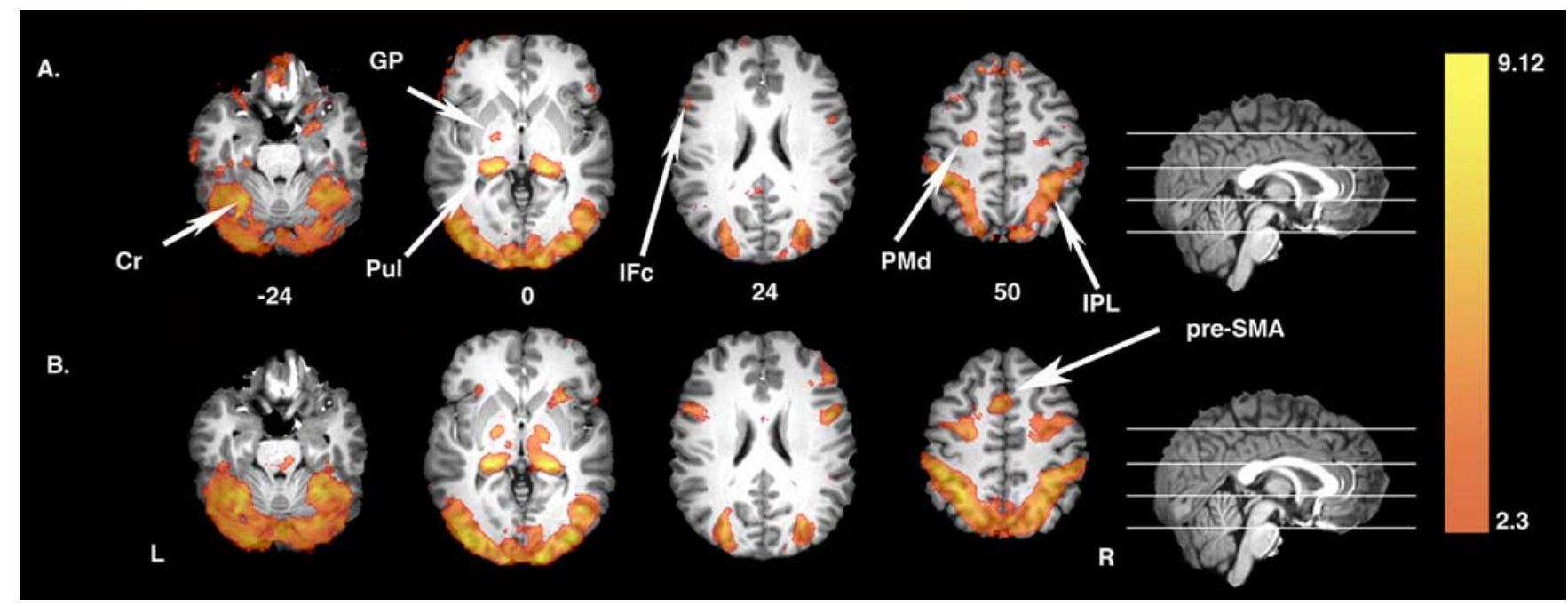

Figure 2. Increased neural activity associated with passive action observation and the intention to learn. $\boldsymbol{A}$, Relative to the resting baseline, passive action observation (Perceptual Control condition) is associated with increased activation in inferior frontal cortex (IFc) and inferior parietal cortex (IPL) of the mirror system as well as in areas associated with motor learning, including bilateral PMd, basal ganglia [globus pallidus (GP)], cerebellum (Cr), and pulvinar nucleus (Pul). B, Similar increases relative to baseline are observed when watching actions with the intention to learn (Learn condition). Note that pre-SMA is only detected in the Learn condition. In this and all figures, colors represent Z-statistic values. L, Left hemisphere; $R$, right hemisphere.

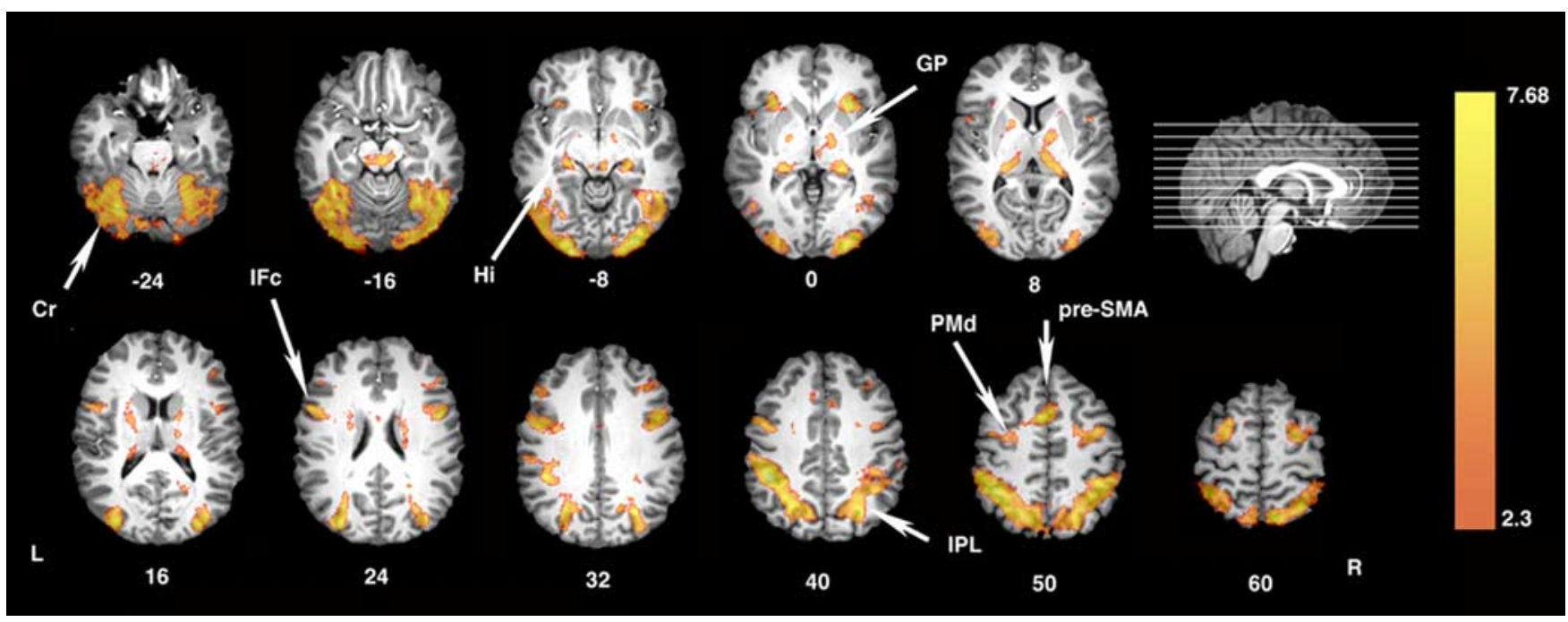

Figure 3. Areas showing increased activation when watching the others' actions with the intention to learn component actions and their sequences. Direct statistical comparison between the Learn and the Perceptual Control conditions reveals bilateral increases throughout the parietofrontal mirror system including bilateral inferior frontal cortex (IFc) and inferior parietal cortex (IPL). Increases are also visible in areas of the sequence learning system including PMd, pre-SMA, basal ganglia [globus pallidus (GP)], cerebellum (Cr), and hippocampus (Hi). L, Left hemisphere; R, right hemisphere.

Among these areas, only responses within a contiguous region of right intraparietal cortex predict the accuracy of subsequent manual performances on the sequential object assembly task (Table 1). As illustrated in Figure 4A, registration to a populationand landmark-based atlas indicates that this region is likely located within and along the banks of the intraparietal sulcus (IPS) and extends rostrally to the post-central sulcus (Van Essen, 2005). Thus, it includes terrain previously identified as a putative homolog of the monkey anterior intraparietal area (AIP) (Binkofski et al., 1998; Culham et al., 2003; Johnson-Frey et al., 2005), and possibly the parietal mirror region of the supramarginal gyrus (Fogassi et al., 2005). Figure $4 B$ shows a strong positive relationship between the individuals' mean activity within this region and their average performance scores on the sequential object assembly task $\left(r_{(18)}=0.72 ; p<0.001\right)$. Although not pictured, reducing the cluster significance threshold to $z=1.6$ (i.e., accepting a less conservative threshold for the size of a clus-
Table 1. Locations of learning-related activation peaks

\begin{tabular}{llll}
\hline$Z$ value & $x$ coordinate $(\mathrm{mm})$ & $y$ coordinate $(\mathrm{mm})$ & $z$ coordinate $(\mathrm{mm})$ \\
\hline 3.46 & 32 & -44 & 28 \\
3.35 & 22 & -50 & 44 \\
3.07 & 22 & -54 & 52 \\
3.03 & 20 & -60 & 56 \\
2.92 & 12 & -62 & 62 \\
2.89 & 6 & -60 & 64
\end{tabular}

Areas are part of a contiguous cluster ( 479 voxels) within and along the right IPS, the activity of which is significantly correlated with a subsequent performance on the object assembly task (clusters determined by thresholding $Z$-statistic images with $Z>2.3$ and a corrected cluster significance threshold of $p<0.05$ ). Values refer to locations relative to the Montreal Neurological Institute standard template brain.

ter of contiguously activated voxels expected by chance) reveals a mostly symmetrical pattern of activity within and along the left IPS, as well as several smaller activations within bilateral regions of the cerebellum and the lateral occipital complex. 

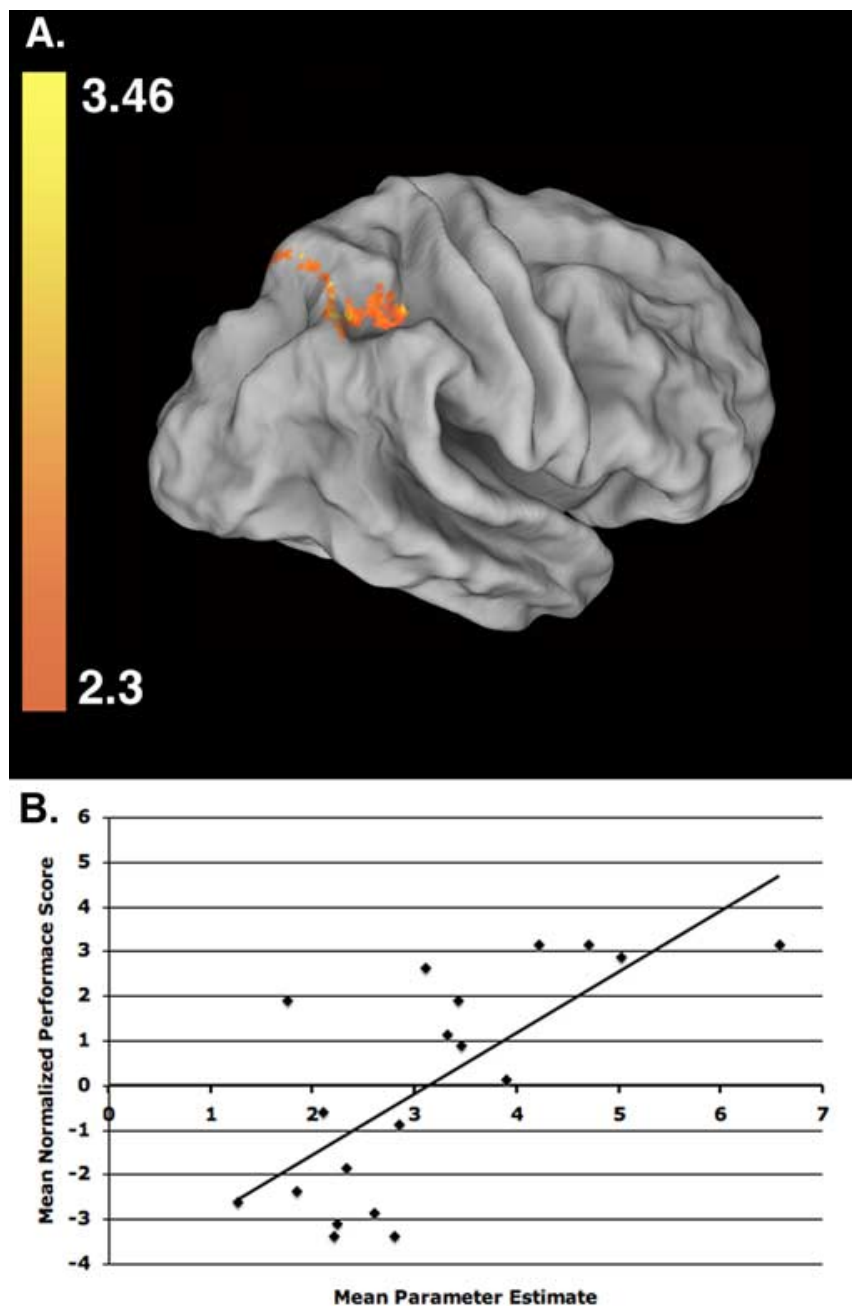

Figure 4. Learning-related activations in right intraparietal cortex. Among regions showing greater activity when attempting to learn observed actions (Learn vs Perceptual Control condition), only activity within and along the right IPS (global peak: $z=3.46 ; p=0.007$; voxels, 479; located at $x=32, y=-44, z=28$ in standard Montreal Neurological Institute space) correlates significantly with subsequent performances on the object assembly task. $A$, This area is shown here on a population-averaged surface rendering that has been partially unfolded to reveal areas within sulci (Van Essen et al., 2001; Van Essen, 2005). B, The correlation between group normalized (mean of 0 ) performance scores for each subject $(n=19)$ on the manual object assembly task versus their mean activity (contrast of parameter estimate values for the Learn condition relative to resting baseline) computed across all voxels within the intraparietal region shown in $\boldsymbol{A}$.

\section{Experiment 2: effects of intending to learn demonstrated component actions for manual reproduction}

Participants in experiment 1 (89.74\%; SE, 0.25\%) and experiment 2 (83\%; SE, 0.29\%) did not differ significantly in the accuracy of the recreated three-dimensional configurations of the objects $\left(t_{(9)}=1.34 ; p=0.21\right)$. This indicates that eliminating the instruction to learn the sequential orders did not affect learning the component actions involved in creating the objects. Participants in experiment $2(72.1 \%$; SE, 3.8\%) were, however, less likely to use the demonstrated sequence when recreating the objects than those in experiment $1\left(89.5 \%\right.$; SE, 3.06\%) $\left(t_{(9)}=5.43\right.$; $p=0.0004)$. Thus, as intended, the instructional manipulation did affect this aspect of performance.

Although not pictured, relative to resting baseline, the effects of both passive observation (Perceptual Control condition) and watching with the intention to learn action components (Learn condition) were strikingly similar to experiment 1 (Fig. 2) [i.e., increased activity bilaterally within the parietofrontal mirror system (i.e., inferior frontal and parietal cortices), PMd, basal ganglia, and cerebellum].

As described for experiment 1, data were again extracted from an ROI defined by the extent of the pre-SMA cluster observed in the Learn condition versus resting baseline contrast. Results are consistent with those reported previously: pre-SMA activity does not differ from baseline during passive observation $\left(t_{(9)}<1.0\right)$. Yet, when observing with the intention to learn, pre-SMA activity is significantly greater than baseline $\left(t_{(18)}=4.5 ; p<0.0015\right)$ and during passive observation $\left(t_{(18)}=3.8 ; p<0.0039\right)$.

Relative to the Perceptual Control condition, Figure 5 shows that observing with the intention to recreate objects using a freely selected procedure again increased activity within the parietofrontal mirror system and in areas associated with motor learning, including cerebellum, basal ganglia, PMd, and pre-SMA.

As shown in Figure 6, a direct comparison between results from experiments 1 and 2 reveals significantly greater activation within these areas when subjects are explicitly instructed to encode the observed procedural sequence. Thus, while these regions do show increased activity when attempting to learn component actions through observation, this modulation is increased with the added demand of encoding the sequence in which component actions are demonstrated.

Finally, in contrast to experiment 1, the relationship between performance of the observed sequence and activity within the right intraparietal cortex ROI defined previously was nonsignificant and trended in the opposite direction $\left(r_{(9)}=-0.55 ; p<\right.$ $0.10)$. This is consistent with the hypothesis that right intraparietal cortex is involved in forming representations of the temporal sequences of observed action components in a manner that supports their subsequent reproduction. By eliminating the requirement to encode and reproduce these sequences, we eliminated the relationship between right intraparietal activity and manual sequence performance.

\section{Discussion}

Recent findings demonstrate that repeated observation of movements can result in experience-dependent changes within M1 (Stefan et al., 2005; Celnik et al., 2006). These changes reflect the acquisition of kinematically precise and effector-specific movement representations and are dependent on input from brain areas involved in coding-observed actions. In contrast, the level of representation acquired in the present experiments is more abstract. Experiments 1 and 2 involve coding the observed component actions or subgoals (e.g., insert short peg into center hole of round disk). In addition, experiment 1 demands representing the temporal sequences in which these components are demonstrated. Neither experiment requires forming representations at a level of precision necessary to reproduce the precise kinematics of the observed movements. Yet, these processes consistently engage brain regions involved in motor representation. Together with previous findings on imitation (Grezes et al., 1998, 1999; Iacoboni et al., 1999; Buccino et al., 2004; Leslie et al., 2004), our results demonstrate that observational learning can induce formation of motor representations at multiple levels of specificity and provide insights into the distributed network of brain areas involved, as well as how they are influenced by the observers' goals. This work may have important implications for developing interventions that seek ways to stimulate and improve motor functions even when the ability for overt practice is absent or limited. 


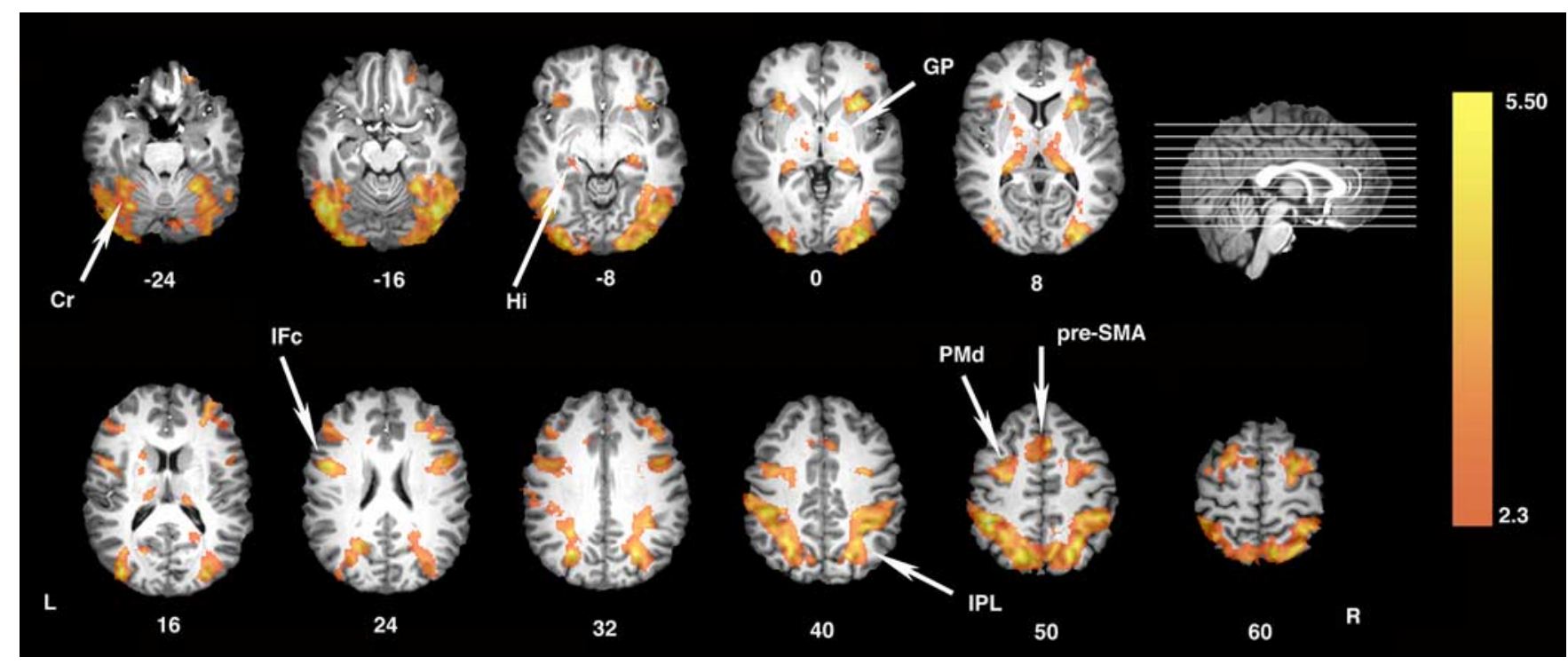

Figure 5. Areas showing increased activation when watching the others' actions with the intention to learn component actions. Similar to experiment 1 (see Fig. 3), direct statistical comparison between the Learn and the Perceptual Control conditions reveals bilateral increases throughout the parietofrontal mirror system including bilateral inferior frontal cortex (IFc) and inferior parietal cortex (IPL). Although participants are not required to learn the demonstrated sequences, increases in activity are still detected in areas of the sequence learning system including PMd, pre-SMA, basal ganglia [globus pallidus (GP)], cerebellum (Cr), and hippocampus (Hi). L, Left hemisphere; R, right hemisphere.

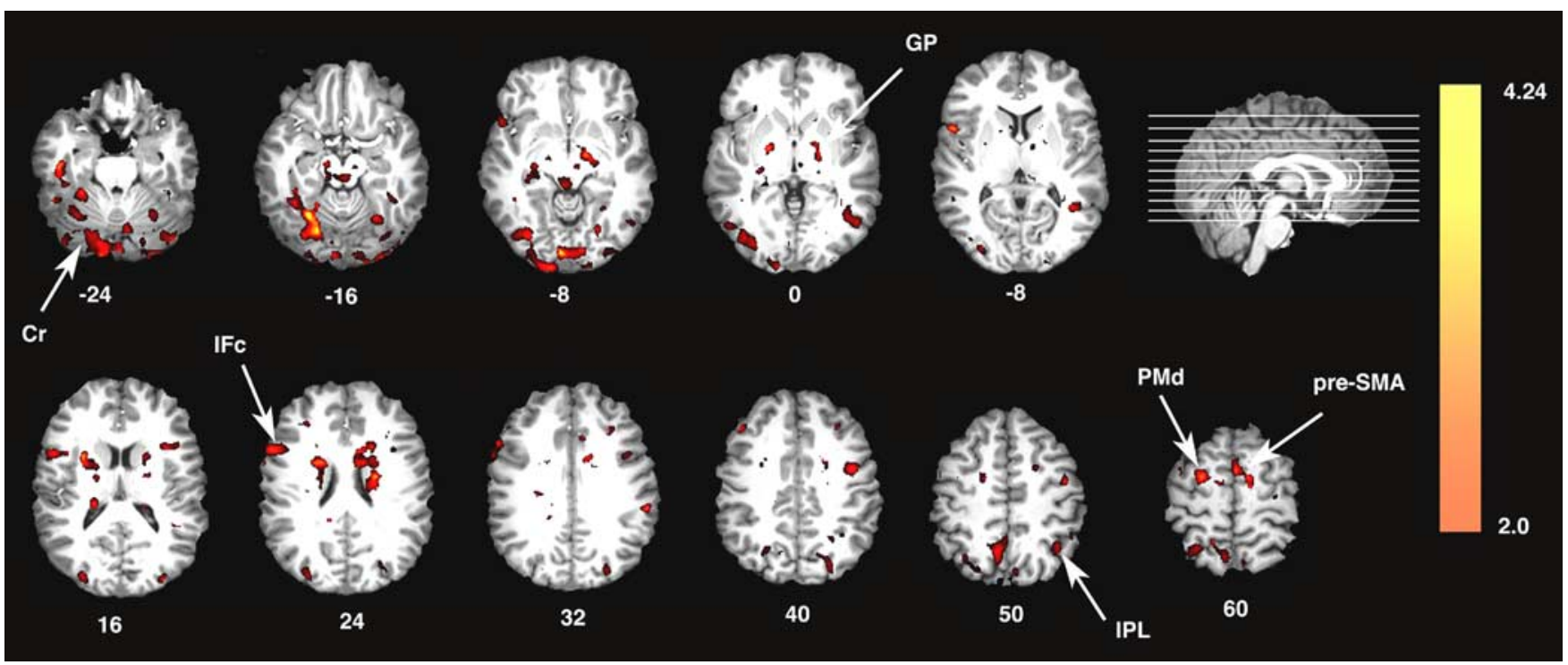

Figure 6. Areas showing increased activity in association with the intention to learn the demonstrated sequence of action components. Direct statistical comparison of experiment 1 versus experiment 2 reveals increased activity in association with the intention to learn the demonstrated sequences throughout the parietofrontal action encoding system [inferior frontal cortex (IFc) and inferior parietal cortex (IPL)] and within areas involved in motor representation, including PMd, pre-SMA, cerebellum (Cr), and basal ganglia [globus pallidus (GP)]. L, Left hemisphere; R, right hemisphere.

Distributed regions activated by passive action observation Relative to the resting baseline, both experiments detected widespread increases in neural activity during passive action observation. These areas included not only the inferior frontal and parietal areas of the mirror system but, unexpectedly, also cortical and subcortical regions known to play a role in motor learning and representation including PMd, cerebellum, and basal ganglia. Whether or not individual neurons within these latter regions can be driven by action observation or execution (i.e., are "mirror" neurons) is a question worthy of investigation.

Likewise, we detect bilateral increases in the pulvinar nucleus of the thalamus. The pulvinar is known to be involved in covert attention (Posner and Petersen, 1990), and it is possible these processes contribute to its engagement here. However, previous studies also demonstrate increased pulvinar activity during motor skill acquisition (Grafton et al., 1992) and when reaches must be directed to remembered target locations (Lacquaniti et al., 1997).

\section{Neuromodulatory effects of observing actions with the} intention to learn

Effects of passive observation

Consistent with previous results (Grezes et al., 1999; Koski et al., 2003; Buccino et al., 2004), we find that activity in inferior frontal and parietal cortices during action observation is increased when participants intend to subsequently reproduce viewed behaviors. This may reflect top-down modulation of existing motor prototypes that represent the component actions or hand-object interactions (Johnson-Frey et al., 2003), from which demonstrated 
behaviors are composed. The present findings extend these neuromodulatory effects to include other regions of the distributed motor representation system, specifically cerebellum, basal ganglia, PMd, and pre-SMA.

Relative to the resting baseline, both experiments only detected significant activity in pre-SMA when participants observed actions with the intention to learn and not during passive action observation. Although care must be taken not to over-interpret a null result, it does appear that pre-SMA is particularly sensitive to the effects of top-down modulation by the observers' intentions to learn observed actions and does not respond to passive action observation. In macaques (Shima and Tanji, 2000) and humans (Sakai et al., 1999), pre-SMA is known to participate in representing the order of movement elements comprising sequential actions. Pre-SMA activity increases when observing sequential visual events with the intention to reproduce them subsequently, or when simply monitoring their accuracy (Schubotz and von Cramon, 2001). Yet, the fact that we observe these differences even when subjects are not instructed to encode observed sequences suggests that pre-SMA is engaged more generally by the intention to learn through observation. Pre-SMA shows increased activity when observers evaluate the accuracy of action sequences being demonstrated by others (Zentgraf et al., 2005), or when asked to infer the intentions behind observed manual actions (Iacoboni et al., 2005). We suggest that pre-SMA activity reflects the filtering of perceived actions in accordance with the observers' intended goals. This is consistent with work relating preSMA function to the more abstract cognitive demands of sensorymotor tasks via its direct connections with areas of prefrontal cortex involved in executive functions (Picard and Strick, 2001).

\section{Effects of sequence encoding}

Many areas within the motor representation network (PMd, cerebellum, basal ganglia, inferior parietal lobule, inferior frontal cortex, and pre-SMA) show increased activity when subjects intend to learn and reproduce both component actions and their demonstrated sequences (experiment 1) versus component actions alone (experiment 2) (Fig. 6). On the one hand, because the overall level of accuracy in recreating the objects did not differ between studies, the additional increase in activity detected in experiment 1 appears related to the demands of encoding and/or internally rehearsing the action sequence rather than nonspecific increases in task difficulty. This is consistent with evidence demonstrating the involvement of these cortical and subcortical structures in motor sequence learning (Doyon et al., 1996; Hazeltine et al., 1997; Grafton et al., 1998). In contrast, results of experiment 2 demonstrate that the intention to encode the observed sequences is not entirely responsible for increased activity within these areas. This is consistent with considerable evidence demonstrating increased activity in these same areas when subjects use motor imagery to learn and/or mentally simulate movements in the absence of overt execution (Decety et al., 1994; Stephan et al., 1995; Grafton et al., 1996; Grezes and Decety, 2001; Johnson et al., 2002).

In humans, there is debate regarding whether the cerebellum contributes to motor sequence learning (Hazeltine and Ivry, 2002) and/or performance (Seidler et al., 2002). Although neither experiment involved immediate motor demands, we detect bilateral increases in cerebellum when passively observing actions. These effects increase when the intention of the observer is to encode component actions for subsequent performance (experiment 2) and are further amplified by the intention to learn the demonstrated sequence (experiment 1 ). Thus, we find a role for the cerebellum in action coding and/or movement simulation apart from motor execution. This is consistent with data from rats showing that ablation of the cerebellum interferes with the ability to learn exploratory behaviors (Morris water maze) observationally but does not affect performance once acquired (Petrosini et al., 2003).

\section{Neural activity correlated with observational learning}

Whereas the modulatory effects of attempting to learn observationally influence many regions, only activity within the IPS predicts the accuracy with which demonstrated sequences of component actions, or subgoals, are subsequently performed. This constitutes a subregion of a much larger set of parietal areas showing increased activity when attempting to encode observed actions for subsequent reproduction (compare Figs. 3, 4A). This relationship is strong evidence for involvement of this region in the formation of representations necessary for successful reproduction of observed action sequences. In both macaques and humans, IPS regions integrate multisensory information into spatial representations for organizing movements of the eyes or forelimbs in peripersonal space (Astafiev et al., 2003; Grefkes and Fink, 2005). Activity near the junction of the IPS and post-central sulcus is a putative homolog of macaque area AIP and is active during visually guided grasping and haptic manipulation (Binkofski et al., 1998; Culham et al., 2003; Johnson-Frey et al., 2005). Involvement of this region here is consistent with previous work implicating rostral inferior parietal cortex in forming representations of manually acquired sequences at a level of abstraction that enables transfer between effectors (Grafton et al., 1998). Moreover, recent findings demonstrate that anterior intraparietal cortex supports goal-dependent representations of both observed (Hamilton and Grafton, 2006) and executed (Tunik et al., 2005) grasping actions.

The asymmetry of this parietal activity is consistent with evidence showing that right-hemisphere injuries affect the ability to discriminate postures visually (Goldenberg, 1999), imitate observed actions with spatial and temporal accuracy (HannaPladdy et al., 2001), and undertake procedures that require multiple subgoals to be attained in specific sequential orders (Hartmann et al., 2005). We conclude that the rostral IPS plays a key role in observational learning of complex action sequences by forming representations of the temporal ordering of component actions that are available to guide subsequent performances of these goal-directed behaviors.

\section{References}

Astafiev SV, Shulman GL, Stanley CM, Snyder AZ, Van Essen DC, Corbetta M (2003) Functional organization of human intraparietal and frontal cortex for attending, looking, and pointing. J Neurosci 23:4689-4699.

Beckmann CF, Jenkinson M, Smith SM (2003) General multilevel linear modeling for group analysis in FMRI. NeuroImage 20:1052-1063.

Binkofski F, Dohle C, Posse S, Stephan KM, Hefter H, Seitz RJ, Freund HJ (1998) Human anterior intraparietal area subserves prehension: a combined lesion and functional MRI activation study. Neurology 50:1253-1259.

Blandin Y (2002) Observational learning: a tool for the acquisition of new motor skills. Ann Psychol 102:523-554.

Buccino G, Vogt S, Ritzl A, Fink GR, Zilles K, Freund HJ, Rizzolatti G (2004) Neural circuits underlying imitation learning of hand actions: an eventrelated fMRI study. Neuron 42:323-334.

Carroll WR, Bandura A (1982) The role of visual monitoring in observational learning of action patterns - making the unobservable observable. J Mot Behav 14:153-167.

Celnik P, Stefan K, Hummel F, Duque J, Classen J, Cohen LG (2006) Encoding a motor memory in the older adult by action observation. NeuroImage 29:677-684.

Cerri G, Shimazu H, Maier MA, Lemon RN (2003) Facilitation from ventral 
premotor cortex of primary motor cortex outputs to macaque hand muscles. J Neurophysiol 90:832-842.

Culham JC, Danckert SL, DeSouza JF, Gati JS, Menon RS, Goodale MA (2003) Visually guided grasping produces fMRI activation in dorsal but not ventral stream brain areas. Exp Brain Res 153:180-189.

Decety J, Perani D, Jeannerod M, Bettinardi V, Tadary B, Woods R, Mazziotta JC, Fazio F (1994) Mapping motor representations with positron emission tomography. Nature 371:600-602.

Doyon J, Owen AM, Petrides M, Sziklas V, Evans AC (1996) Functional anatomy of visuomotor skill learning in human subjects examined with positron emission tomography. Eur J Neurosci 8:637-648.

Duvernoy HM (1991) The human brain: surface, blood supply, and threedimensional sectional anatomy, Ed 2. New York: Springer Wien.

Fogassi L, Ferrari PF, Gesierich B, Rozzi S, Chersi F, Rizzolatti G (2005) Parietal lobe: from action organization to intention understanding. Science 308:662-667.

Gallese V, Fadiga L, Fogassi L, Rizzolatti G (1996) Action recognition in the premotor cortex. Brain 119:593-609.

Goldenberg G (1999) Matching and imitation of hand and finger posturesin patients with damage in the left or right hemispheres. Neuropsychologia 37:559-566.

Grafton ST, Mazziotta JC, Presty S, Friston KJ, Frackowiak RS, Phelps ME (1992) Functional anatomy of human procedural learning determined with regional cerebral blood flow and PET. J Neurosci 12:2542-2548.

Grafton ST, Arbib MA, Fadiga L, Rizzolatti G (1996) Localization of grasp representations in humans by positron emission tomography. 2. Observation compared with imagination. Exp Brain Res 112:103-111.

Grafton ST, Hazeltine E, Ivry RB (1998) Abstract and effector-specific representations of motor sequences identified with PET. J Neurosci 18:9420-9428.

Grefkes C, Fink GR (2005) The functional organization of the intraparietal sulcus in humans and monkeys. J Anat 207:3-17.

Grezes J, Decety J (2001) Functional anatomy of execution, mental simulation, observation, and verb generation of actions: a meta-analysis. Hum Brain Mapp 12:1-19.

Grezes J, Costes N, Decety J (1998) Top down effect of the strategy to imitate on the brain areas engaged in perception of biological motion: a PET study. Cogn Neuropsychol 15:553-582.

Grezes J, Costes N, Decety J (1999) The effects of learning and intention on the neural network involved in the perception of meaningless actions. Brain 122:1875-1887.

Hamilton AF, Grafton ST (2006) Goal representation in human anterior intraparietal sulcus. J Neurosci 26:1133-1137.

Hanna-Pladdy B, Daniels SK, Fieselman MA, Thompson K, Vasterling JJ, Heilman KM, Foundas AL (2001) Praxis lateralization: errors in right and left hemisphere stroke. Cortex 37:219-230.

Hartmann K, Goldenberg G, Daumuller M, Hermsdorfer J (2005) It takes the whole brain to make a cup of coffee: the neuropsychology of naturalistic actions involving technical devices. Neuropsychologia 43:625-637.

Hazeltine E, Ivry RB (2002) Neuroscience. Can we teach the cerebellum new tricks? Science 296:1979-1980.

Hazeltine E, Grafton ST, Ivry R (1997) Attention and stimulus characteristics determine the locus of motor-sequence encoding. A PET study. Brain 120:123-140.

Hermsdörfer J, Goldenberg G, Wachsmuth C, Conrad B, Ceballos-Baumann AO, Bartenstein P, Schwaiger M, Boecker H (2001) Cortical correlates of gesture processing: clues to the cerebral mechanisms underlying apraxia during the imitation of meaningless gestures. NeuroImage 14:149-161.

Heyes CM, Foster CL (2002) Motor learning by observation: evidence from a serial reaction time task. Q J Exp Psychol A 55:593-607.

Iacoboni M, Woods RP, Brass M, Bekkering H, Mazziotta JC, Rizzolatti G (1999) Cortical mechanisms of human imitation. Science 286:2526-2528.

Iacoboni M, Molnar-Szakacs I, Gallese V, Buccino G, Mazziotta JC, Rizzolatti G (2005) Grasping the intentions of others with one's own mirror neuron system. PLoS Biol 3:e79.

Jenkinson M, Smith S (2001) A global optimisation method for robust affine registration of brain images. Med Image Anal 5:143-156.

Jenkinson M, Bannister P, Brady M, Smith S (2002) Improved optimization for the robust and accurate linear registration and motion correction of brain images. NeuroImage 17:825-841.

Johnson SH, Rotte M, Grafton ST, Hinrichs H, Gazzaniga MS, Heinze H-J (2002) Selective activation of a parieto-frontal circuit during implicity imagined prehension. NeuroImage 17:1693-1704.
Johnson-Frey SH, Maloof FR, Newman-Norlund R, Farrer C, Inati S, Grafton SG (2003) Actions or hand-object interactions? Human inferior frontal cortex and action observation. Neuron 39:1053-1058.

Johnson-Frey SH, Vinton D, Norlund RN, Grafton SG (2005) Cortical topography of the human anterior intraparietal area. Cognit Brain Res 23:397-405.

Koski L, Iacoboni M, Dubeau MC, Woods RP, Mazziotta JC (2003) Modulation of cortical activity during different imitative behaviors. J Neurophysiol 89:460-471.

Lacquaniti F, Perani D, Guigon E, Bettinardi V, Carrozzo M, Grassi F, Rossetti Y, Fazio F (1997) Visuomotor transformations for reaching to memorized targets: a PET study. NeuroImage 5:129-146.

Lashley KS (1951) The problem of serial order in behavior. In: The Hixon Symposium (Jeffress LA, ed), pp 112-146. Chichester, UK: Wiley.

Leslie KR, Johnson-Frey SH, Grafton ST (2004) Functional imaging of face and hand imitation: towards a motor theory of empathy. NeuroImage 21:601-607.

Oldfield RC (1971) The assessment and analysis of handedness: the Edinburgh inventory. Neuropsychologia 9:97-113.

Petrosini L, Graziano A, Mandolesi L, Neri P, Molinari M, Leggio MG (2003) Watch how to do it! New advances in learning by observation. Brain Res Brain Res Rev 42:252-264.

Picard N, Strick PL (2001) Imaging the premotor areas. Curr Opin Neurobiol 11:663-672.

Posner MI, Petersen SE (1990) The attention system of the human brain. Annu Rev Neurosci 13:25-42.

Rizzolatti G, Craighero L (2004) The mirror-neuron system. Annu Rev Neurosci 27:169-192.

Rizzolatti G, Camarda R, Fogassi L, Gentilucci M, Luppino G, Matelli M (1988) Functional organization of inferior area 6 in the macaque monkey. II. Area F5 and the control of distal movements. Exp Brain Res 71:491-507.

Sakai K, Hikosaka O, Miyauchi S, Sasaki Y, Fujimaki N, Putz B (1999) Presupplementary motor area activation during sequence learning reflects visuo-motor association. J Neurosci 19: RC1(1-6).

Schendan HE, Searl MM, Melrose RJ, Stern CE (2003) An FMRI study of the role of the medial temporal lobe in implicit and explicit sequence learning. Neuron 37:1013-1025.

Schubotz RI, von Cramon DY (2001) Interval and ordinal properties of sequences are associated with distinct premotor areas. Cereb Cortex 11:210-222.

Seidler RD, Purushotham A, Kim SG, Ugurbil K, Willingham D, Ashe J (2002) Cerebellum activation associated with performance change but not motor learning. Science 296:2043-2046.

Shima K, Tanji J (2000) Neuronal activity in the supplementary and presupplementary motor areas for temporal organization of multiple movements. J Neurophysiol 84:2148-2160.

Smith SM (2002) Fast robust automated brain extraction. Hum Brain Mapp 17:143-155.

Stefan K, Cohen LG, Duque J, Mazzocchio R, Celnik P, Sawaki L, Ungerleider L, Classen J (2005) Formation of a motor memory by action observation. J Neurosci 25:9339-9346.

Stephan KM, Fink GR, Passingham RE, Silbersweig D, Ceballos-Baumann AO, Frith CD, Frackowiak RS (1995) Functional anatomy of the mental representation of upper extremity movements in healthy subjects. J Neurophysiol 73:373-386.

Tunik E, Frey SH, Grafton ST (2005) Virtual lesions of the human anterior intraparietal area disrupt goal-dependent on-line adjustments of grasp. Nat Neurosci 8:505-511.

Van Essen DC (2005) A population-average, landmark- and surface-based (PALS) atlas of human cerebral cortex. NeuroImage 28:635-662.

Van Essen DC, Drury HA, Dickson J, Harwell J, Hanlon D, Anderson CH (2001) An integrated software suite for surface-based analyses of cerebral cortex. J Am Med Inform Assoc 8:443-459.

Woolrich MW, Ripley BD, Brady M, Smith SM (2001) Temporal autocorrelation in univariate linear modeling of FMRI data. NeuroImage 14:1370-1386.

Worsley KJ, Evans AC, Marrett S, Neelin P (1992) A three-dimensional statistical analysis for CBF activation studies in human brain. J Cereb Blood Flow Metab 12:900-918.

Zentgraf K, Stark R, Reiser M, Kunzell S, Schienle A, Kirsch P, Walter B, Vaitl D, Munzert J (2005) Differential activation of pre-SMA and SMA proper during action observation: effects of instructions. NeuroImage 26:662-672. 